\title{
Influence of Thromboelastometry in making clinical decisions in a patient with an intra aortic balloon pump and severe Thrombocytopenia
}

\author{
Gómez Ana Mº, Paniagua Pilar, Rivilla Ma Teresa, Moral Victoria \\ Hospital de la Santa Creu I Sant Pau. Barcelona, Spain
}

\section{Background}

Haemorrhage is a significant risk during the Intra Aortic Balloon Pump (IABP) removal, with an increased risk in patients with Thromobocytopenia. However, the platelet count should not be the sole consideration in whether or not to remove the IABP before transfusion. Viscoelastic tests can provide a wider perspective about the patient's capacity to form a clot, despite the platelet count.

\section{Case Report}

A woman in her 60 s with a history of hypertension, mellitus diabetes, and atrial fibrillation Surgery: Mechanical aortic valve replacement due to severe stenosis.

Complications: - Intraoperative isquemia that caused cardiac arrest $\rightarrow$ bypass to the left anterior descending coronary artery. - Severe bleeding presented upon intensive care admission, requiring reintervention.

- Cardiogenic shock associated with akinesia of the anterior wall and a new severe tricuspid insufficiency

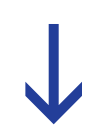

Emergency cardiac catheterization $\rightarrow$ left coronary sinus disection that mildly affected the ostium of the common trunk, without compromising the flow $\rightarrow$ Implantation of the IABP.

The patient improved from the cardiogenic shock during the following 6 days, allowing for the removal of the IABP.

* Normal partial thromboplastin time and prothrombin time

* Moderate thrombocytopenia (37x10E9/L) - Secondary to the device?

- Heparin-induced thrombocytopenia.

Not transfuse platelets + study the clot formation with Tromboelastometry.
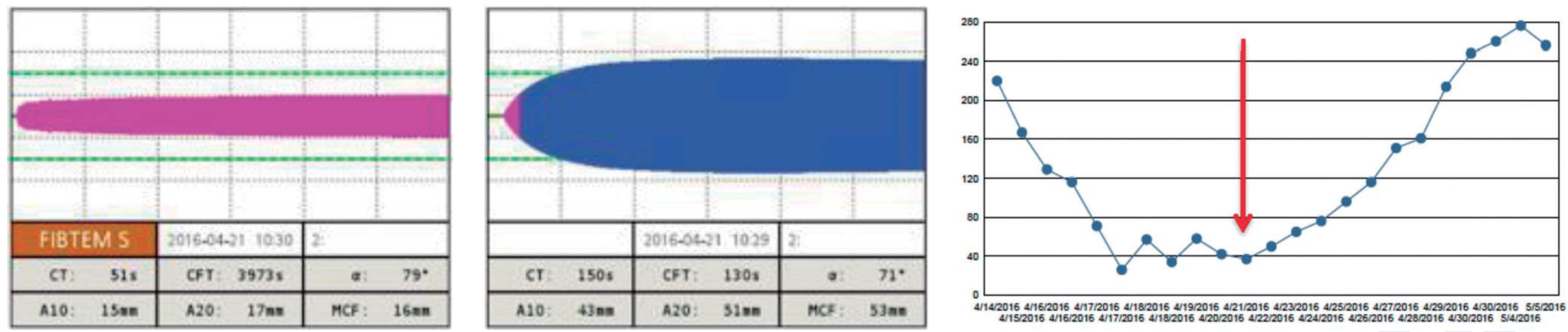

Removal of the IABP, without platelet transfusion. The patient did not have any complications, during or after the procedure. The heparin-induced thrombocytopenia was ruled out and the platelets gradually increased.

\section{Conclusions}

Thrombocytopenia is a significant risk factor for bleeding during the IABP removal, and therefore platelet transfusion prior to the procedure must be considered. However, the transfusion is not without risks, particularly if heparin-induced thrombocytopenia is suspected. Thromboelastometry may be able to predict more accurately the bleeding tendency, rather than the platelet count as an isolated test. This is due to Thromboelastometry being a global assay that determines whole blood clot formation, thus helping to decide whether or not to transfuse prior to an invasive procedure. 